\title{
Magnetooptic Effects in the Photonic Crystal Fiber as a Function of the Polarization State
}

\author{
K. BARCZAK* \\ Department of Optoelectronics, Faculty of Electrical Engineering at the Silesian University of Technology \\ Akademicka 2, 44-100 Gliwice, Poland
}

\begin{abstract}
In optical fibers there exists an optical birefringence induced by external factors and stress produced in technological processes. The induced birefringence makes difficult to apply optical fibers in heads of polarimetric sensors. It has been proposed to use a photonic crystal fiber working in an index guiding regime due to the small value of stress in a fiber core produced by internal and external factors. This article presents an expansion of our earlier investigations. The expansion consists of investigations concerning the influence of external magnetic field induction on the state of polarization of light in the function of an azimuth of the input linear polarization $\phi$. On the basis of the resulting characteristics optimal working points of the tested fiber were determined.
\end{abstract}

DOI: 10.12693 /APhysPolA.124.384

PACS: 42.25.Bs, 42.79.Pw, 33.20.Kf, 42.70.-a

\section{Introduction}

In recent years, intensive investigations concerning elaborations of optical sensors of various physical effects are performed [1-8]. Optical fibers may be applied in the technique of measuring electric currents and magnetic fields. In such measurements the optical birefringence is applied $[9,10]$. Induced linear birefringence of the optical fiber results from two factors. The first one is stress produced in technological processes $[11,12]$. This stress cannot be avoided. The second, additional birefringence, induced by deformation, is produced by external forces. This deformation creates a new stress and changes the stress distribution produced in the technological process. Relaxation processes can take a long time or cannot lead to equilibrium state. Fluctuations of stress distribution result from a small value of their activation energy. The main factor, which produces these fluctuations, are temperature fluctuations. Summarizing, the optical fibers do not, generally, maintain polarization because the state of polarization is still changing.

For these reasons, it is very important to investigate how the induced birefringence affects the Faraday effect. This dependence cannot be achieved on the basis of theoretical analysis. For this reason investigation must be based merely on measurement results. In the article [10] the author presented the influence of external disturbances on the state of polarization. The similar measurements will be presented in this article but for azimuth of input linear polarization ranging from 0 to 180 degrees.

The motivation of the presented paper is to verify the following thesis: "An optical fiber current sensor based on photonic crystal fiber can operate with a relatively small linear birefringence induced by deformation".

*e-mail: Kamil.Barczak@polsl.pl
The presented results were obtained using the same test stand as the one presented in the article [13]. The author modified only the input section by introducing the special FC connector described in the next section (Fig. 1). All measurements concern a constant magnetic induction (DC mode).

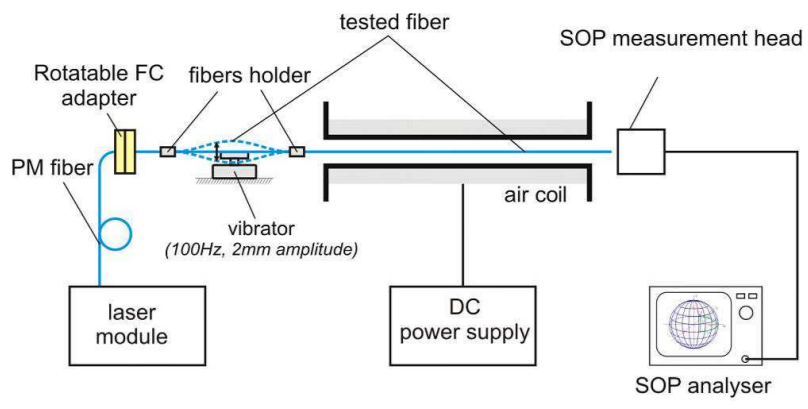

Fig. 1. Measurement stand.

\section{Experimental stand}

The object of the research is a commercial photonic crystal fiber with a glass core (index guiding), characterized by its single-mode operation at a wavelength of $\lambda=650 \mathrm{~nm}$ (the same as in the article [13]). The attenuation at this wavelength is less than $15 \mathrm{~dB} / \mathrm{km}$.

The measurement stand was equipped with a special rotatable FC adapter (Fig. 2). The laser diode was pigtailed with a polarization maintaining fiber and equipped with an FC connector. This connector was inserted into the rotatable adapter which can be rotated thanks to a special construction of the adapter. Rotation of the PM fiber produces the same rotation angle of linear polarization of the light emerging from the fiber output.

The source of the magnetic field was an air-core coil $80 \mathrm{~cm}$ long. The coil was supplied from DC laboratory 
power supply. A characteristic part of the stand is a vibrator which can vibrate with an amplitude of $2 \mathrm{~mm}$ and a frequency of $100 \mathrm{~Hz}$.

The schematic view of described measurement stand with the special rotatable FC adapter is presented in Fig. 1.

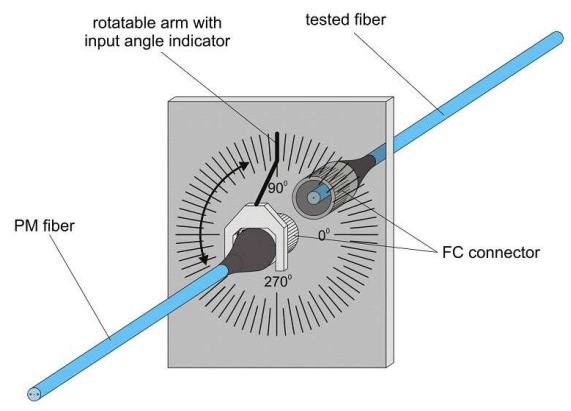

Fig. 2. Special rotatable FC adapter.

\section{Experimental results}

Linear birefringence has a very high influence on the state of polarization at the output of the fiber [11]. For this reason the magnetooptic Faraday effect significantly depends on a linear birefringence. Finally, the Verdet constant depends on the linear birefringence of the fiber because the twist of azimuth of the state of polarization depends on birefringence, too. According to the basic polarization theory, this behavior is due to the ellipticity of

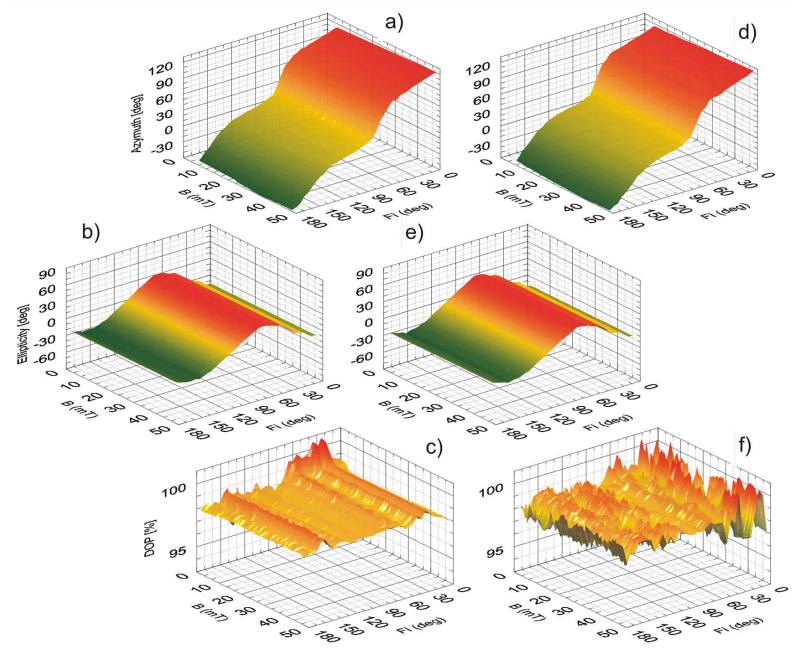

Fig. 3. Characteristics of: azimuth as a function of azimuth of input linear polarization and magnetic induction (a) without vibration, (d) with vibration; ellipticity as a function of azimuth of input linear polarization and magnetic induction (b) without vibration, (e) with vibration; $D O P$ as a function of azimuth of input linear polarization and magnetic induction (c) without vibration, (f) with vibration.

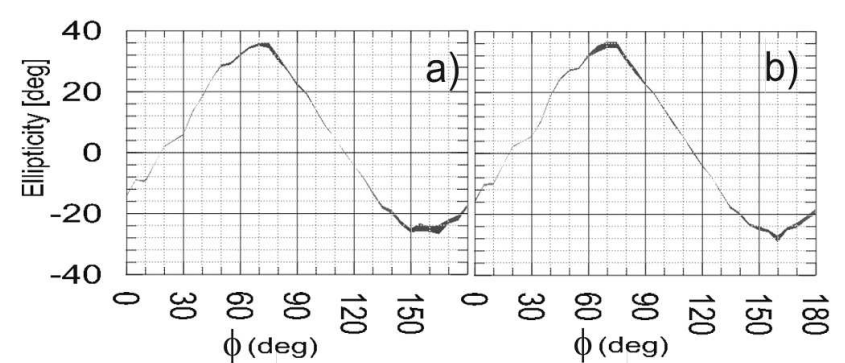

Fig. 4. Characteristics (projection from Fig. $3 \mathrm{~b}$ and e) of ellipticity as a function of azimuth of input linear polarization: (a) without vibration, (d) with vibration.

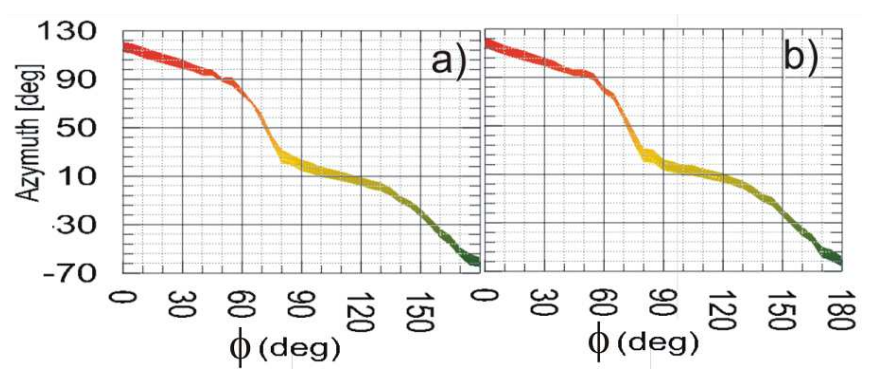

Fig. 5. Graphs (projection from Fig. 3a and d) of azimuth as a function of azimuth of input linear polarization: (a) without vibration, (b) with vibration.

SOP produced in linear birefringence. This birefringence is produced by the elastooptic effect. Figure 3 shows the azimuth, ellipticity and degree of polarization (DOP) versus the azimuth of input linear polarization and magnetic induction. In Fig. 3a and d we can see how the magnetooptic effect (azimuth vs. magnetic induction $\boldsymbol{B}$ ) depends on the azimuth of input linear polarization. These results comply with theory [11]. The observed dependence of ellipticity coincides with the same theory Fig. $3 \mathrm{~b}$ and e. The most important aspects can be seen in the projection illustrated in Fig. 4 and Fig. 5. Especially the ellipticity $-\phi$ projection (azimuth of input linear polarization) showed the region where the ellipticity is about zero (Fig. 5). In both cases (without and with vibration) these are the same two regions: (1) $\phi$ between 10 and 20 degrees; (2) $\phi$ between 110 and 120 degrees. In the same regions, the relatively small dependence of $\phi$ is observed on the azimuth projection (Fig. 4). For this reason the azimuth is more stable. Summarizing, these regions are optimal in the case of applying an optical fiber as the sensor head, especially as a polarimetric sensor head with linear polarimeter configuration (in this case the zero value of ellipticity is optimal).

Calculated values of the Verdet constant depending on the azimuth of input linear polarization $\phi$ are shown in Fig. 6 and Fig. 7. The aforesaid regions are marked in this graphs. Small dependences on $\phi$ in these regions are to be observed. These results confirm better the stability of the azimuth. 


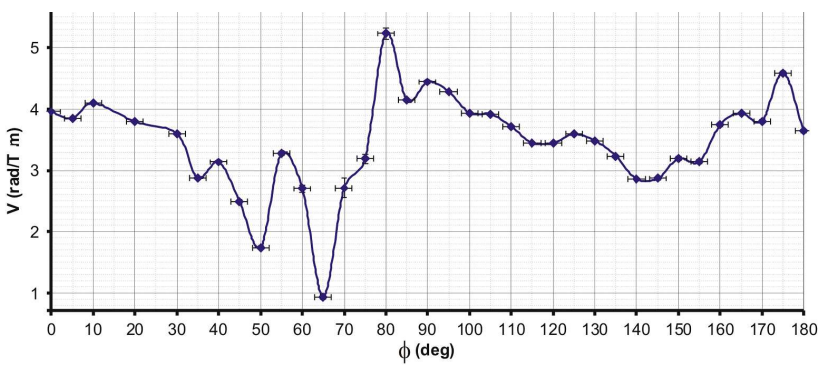

Fig. 6. Characteristics of the Verdet constant as a function of azimuth of input linear polarization (without vibration).

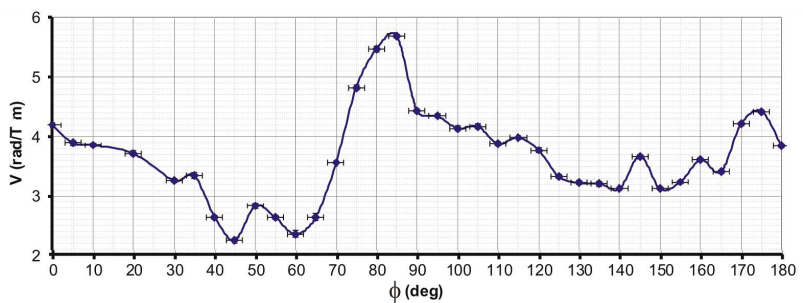

Fig. 7. Characteristics of the Verdet constant as a function of azimuth of input linear polarization (with vibration).

It is also noteworthy that the DOP is very stable. The DOP does not extend beyond the range from $95 \%$ to $100 \%$. This situation is presented in the parts $\mathrm{c}$ and $\mathrm{f}$ in Fig. 3. The very high quantities of DOP confirm that this fiber has small linear birefringence induced by stress [12]. The same conclusion is valid for the vibration case - Fig. 3f.

The small differences between measurements registered without vibration and those registered in the vibration mode are very promising (Fig. 3).

\section{Conclusions}

The performed measurements confirmed that photonic crystal fibers (PCF) display rather good polarimetric properties for application in polarimetric sensors. First of all, it has been shown that optical fibers characterized by low birefringence also have as good magnetooptic properties as an isotropic medium, conditioned by a proper setting of the operating point, which should be characterized by the following features: ellipticity close to zero, small variations of the Verdet constant in the function of azimuth of input polarization state, ellipticity close to zero increases in the function of magnetic field induction.

\section{References}

[1] T. Pustelny, J. Ignac-Nowicka, Z. Opilski, Opt. Appl. 34, 563 (2004).

[2] K. Gut, D. Nabaglo, Acta Phys. Pol. A 116, 307 (2009).

[3] K. Barczak, T. Pustelny, Z. Zycki, T. Blazejczyk, Acta Phys. Pol. A 116, 250 (2009).

[4] J.J. Hu, Y. Wang, J.L. Lim, T. Zhang, K.B. Milenko, Z. Chen, M. Jiang, T. Wolinski, IEEE Sensor J. 12, 1239 (2012).

[5] K. Barczak, T. Pustelny, D. Dorosz, J. Dorosz, Europ. Phys. J. Spec. Top. 154, 11 (2008).

[6] E. Maciak, Z. Opilski, T. Pustelny, M. Bednorz, J. Phys. IV: JP (France) 129, 131 (2005).

[7] T. Pustelny, J. Ignac-Nowicka, Z. Opilski, Opt. Appl. 34, 249 (2004).

[8] K. Gut, K. Nowak, Europ. Phys. J. Spec. Top. 154, 89 (2008).

[9] T. Pustelny, K. Barczak, K. Gut, J. Wojcik, Opt. Appl. 34, 531 (2004).

[10] K. Barczak, Bull. Pol. Acad. Sci., Techn. Sci. 59, 409 (2011).

[11] K. Barczak, T. Pustelny, D. Dorosz, J. Dorosz, Acta Phys. Pol. A 118, 1087 (2010).

[12] A.W. Domański, Opto-Electron. Rev. 13, 171 (2005).

[13] K. Barczak, Acta Phys. Pol. A 122, 793 (2012). 